\title{
UNA HERRAMIENTA METODOLÓGICA PARA EL APRENDIZAJE DE LA ESTADÍSTICA Y LA ATENCIÓN A LA DIVERSIDAD
}

\author{
Jesús Murillo Ramón \\ Roberto Castellanos Fonseca \\ Universidad de La Rioja
}

\begin{abstract}
RESUMEN
El objetivo de nuestra investigación es construir, ejemplificar y analizar los beneficios de una herramienta metodológica pensada para mejorar el éxito escolar en el aprendizaje de la Estadística en la educación secundaria obligatoria, trabajando con alumnos de diversificación curricular. En estos momentos hemos construido el sistema tutorial y hemos realizado una evaluación a través del test de BADYG renovado y una estadística de rendimientos mediante pruebas de control inicial y final. Con la información que estamos recogiendo de los procesos y resolución de las actividades desarrolladas en el sistema tutorial diseñado, pretendemos construir los instrumentos de análisis correspondientes y proceder al estudio de casos y análisis.
\end{abstract}

PALABRAS CLAVE: Estadística, aprendizaje, entorno interactivo, atención a la diversidad, hoja de cálculo, Internet.

\section{ABSTRACT}

The aim of our research is to build, exemplify and analyze the benefits of a methodological tool thought for improving the success of the students when learning Statistics in the compulsory secondary education, working with pupils belonging to a diversification group. For the time being we have built the tutorial system and achieved an assessment through the updated BADYG test and a statistical analysis of the students outputs based on initial and final control tests. With the information we are collecting of the processes and resolution of the activities developed in the designed tutorial system, we expect to build the corresponding analysis tools and proceed with the respective study of cases and analysis.

KEY WORDS: Statistics, learning, interactive environment, attention to diversity, spreadsheet, Internet. 


\section{Introducción}

Todos los informes sobre el uso de Internet, coinciden en señalar su espectacular incremento de año en año. Es pues un hecho que vivir, estudiar y trabajar ajenos a Internet no es posible. "Estamos condenados a ser modernos. No podemos prescindir de la ciencia y de la tecnología", (Octavio Paz). La incorporación de las Tecnologías de la Información y de la Comunicación (TIC) en todos los sectores sociales ha hecho cambiar los planteamientos actuales de la enseñanza tanto en la vertiente escolar como en la empresarial puesto que Internet además de ser un lugar donde transmitir contenidos es sobre todo un medio con un gran potencial educativo en sí mismo, puesto que aúna la oportunidad de conjugar elementos multimedia (textos, imágenes, sonido) a la vez que incorpora interactividad y personalización. Se trata de aprovechar los nuevos usos tecnológicos para mejorar la enseñanza y el aprendizaje de las matemáticas en todos los niveles educativos.

Por otra parte, parece claro que en la sociedad actual, para los momentos presentes y futuros, las exigencias de formación inicial y profesional para desenvolverse en los distintos entornos, son bastante diferentes de las necesarias no hace mucho tiempo. La toma de decisiones requiere comprender, modificar y producir mensajes de todo tipo, y en la información que se maneja, cada vez aparecen más tablas, gráficos y expresiones que exigen conocimientos matemáticos y estadísticos para su correcta interpretación. Las destrezas básicas de comunicación están adquiriendo un papel primordial. En nuestra sociedad actual basada en la información, resulta indispensable que los alumnos aprendan a comunicarse matemáticamente, que tengan oportunidad de leer, escribir y discutir ideas para las que el uso del lenguaje matemático sea algo natural. Además los ciudadanos deben estar preparados para adaptarse a los continuos cambios que se generan. En un entorno de cambio tan acelerado, es conveniente dejar de preparar individuos para desempeñar una única ocupación y realizar una formación en el aprendizaje. Es decir, se trata de preparar a las personas para poder aprender y saber comunicar.

La presencia de la estadística en los medios de comunicación y el uso que se hace en muy diversos ámbitos y materias plantea la necesidad que en la actualidad tenemos de promover una enseñanza efectiva que capacite a los futuros ciudadanos para analizar de forma crítica las presentaciones e interpretaciones, muchas veces sesgadas, que contiene la información de naturaleza estadística. Además de su carácter instrumental para otras disciplinas, se reconoce el valor del desarrollo del razonamiento estadístico en una sociedad caracterizada por la disponibilidad de información y la necesidad de toma de decisiones en ambiente de incertidumbre (Batanero, 2002).

\section{Situación e interés}

En los países democráticos donde las decisiones políticas y sociales implican aspectos técnicos cada vez más complejos, es imprescindible que exista un electorado culto y bien informado. Cada vez está más asumido que la dinámica del mundo moderno exige que todo ciudadano, para ejercer sus derechos y comprender su entorno, posea una cierta alfabetización en estadística.

El objetivo principal sería proporcionar una cultura estadística, de manera que los alumnos sean capaces de interpretar y evaluar de manera crítica la información estadística que aparece en los medios de comunicación y capacitarlos para discutir o comunicar sus opiniones al respecto. Nuestros estudiantes deben comprender la 
Estadística lo suficientemente bien para "consumir" adecuadamente la información con la que somos bombardeados diariamente, piensen críticamente sobre ella, y sean capaces de tomar decisiones correctas basadas en esta información. Debemos conseguir la "alfabetización estadística". Según Gal (citado por Rumsey, 2002) "la habilidad de la gente para interpretar y evaluar críticamente información estadística y argumentos basados en datos que aparecen en diversos canales mediáticos, y su habilidad para discutir sus opiniones teniendo en cuenta tal información estadística" o siguiendo a Garfield "la comprensión del lenguaje estadístico: palabras, símbolos y términos. Ser capaz de interpretar gráficos y tablas. Ser capaz de leer y entender las noticias que aparecen en las noticias, medios de comunicación, encuestas,.." (citado por Rumsey, 2002)

El interés de nuestras autoridades educativas por la alfabetización estadística, en cuanto a conseguir la competencia estadística y la formación en las TIC de nuestros alumnos de Educación Secundaria, se muestra claramente en el decreto de mínimos de la ESO (RD1631/2006, 2007). Como objetivo plantea que nuestros alumnos sean capaces de: emplear los métodos y procedimientos estadísticos y probabilísticos para enjuiciar la realidad o las informaciones que de ella ofrecen los medios de comunicación, la publicidad, Internet u otras fuentes de información; analizar críticamente la función que desempeñan y valorar su aportación para una mejor comprensión de los mensajes.

Como competencia básica: esta competencia consiste en disponer de habilidades para buscar, obtener, procesar y comunicar información, y para transformarla en conocimiento. Incorpora diferentes habilidades, que van desde el acceso a la información hasta su transmisión en distintos soportes una vez tratada, incluyendo la utilización de las tecnologías de la información y la comunicación como elemento esencial para informarse, aprender y comunicarse. Disponer de información no produce de forma automática conocimiento. Significa, asimismo, comunicar la información y los conocimientos adquiridos empleando recursos expresivos que incorporen, no sólo diferentes lenguajes y técnicas específicas, sino también las posibilidades que ofrecen las tecnologías de la información y la comunicación.

Trabajando el currículo de Estadística con los alumnos de Educación Secundaria, podemos utilizar algunas herramientas, cuyo uso adecuado podría suponer mejoras en el aprendizaje, a la vez que eliminamos cálculos tediosos y poco constructivos. El análisis y la organización de la información se facilita en gran manera con las Hojas de Cálculo, que además posibilitan la utilización de gráficos sencillos, el tratamiento de gran cantidad de datos y una economía de esfuerzos, en cuanto a que permite automatizar los cálculos y realizarlos con gran rapidez, pudiendo determinar de forma cómoda las medidas más representativas de las distribuciones estadísticas. Los cálculos se pueden rehacer de una forma muy rápida cuando se modifican los datos, de esta manera se consigue que las acciones mecánicas y más repetitivas se realicen de una forma ágil y poco tediosa, liberando tiempo para dedicarlo a aspectos más formativos como la formulación de preguntas y la comprensión de conceptos e ideas.

La revolución producida por las TIC sobre la sociedad, impacta en particular sobre los sistemas educativos; definiendo nuevas necesidades como por ejemplo la de formar ciudadanos con conocimiento sobre estas nuevas tecnologías, y con capacidad de reflexión y crítica (usuarios autónomos). Estas nuevas necesidades, conllevan un replanteamiento del concepto de formación general e incluso el de persona 
alfabetizada. Por otra parte, la Educación Matemática plantea nuevas necesidades para este siglo; poniendo el acento en el desarrollo de ciertas competencias por parte de los alumnos. Aceptar estos retos supone un cambio conceptual en la organización de las enseñanzas obligatorias para adaptarse a los modelos de formación más centrados en el aprendizaje, es decir, en el estudiante y en su trabajo, y que les permitan "aprender a aprender". En este nuevo escenario educativo que ha aparecido en la llamada Sociedad de la Información con la llegada de las TIC, se genera una forma de planificar y elaborar actividades y desarrollar proyectos en el proceso de enseñanza aprendizaje utilizando estas herramientas, que enriquecen el proceso formativo individual. Ser competente en la utilización de las tecnologías de la información y la comunicación como instrumento de trabajo intelectual incluye utilizarlas en su doble función de transmisoras y generadoras de información y conocimiento. Se utilizarán en su función generadora al emplearlas, por ejemplo, como herramienta en el uso de modelos de procesos matemáticos, físicos, sociales, económicos o artísticos. Asimismo, esta competencia permite procesar y gestionar adecuadamente información abundante y compleja, resolver problemas reales, tomar decisiones, trabajar en entornos colaborativos ampliando los entornos de comunicación para participar en comunidades de aprendizaje formales e informales, y generar producciones responsables y creativas. (RD1631/2006, 2007)

En estos momentos, parece claro para casi todo el mundo que las TIC y los recursos informáticos son herramientas que promueven un aprendizaje activo de los alumnos, y que su uso es parte integrante de la vida cotidiana. La incorporación de herramientas tecnológicas como recurso didáctico para el aprendizaje y para la resolución de problemas, contribuye a mejorar la competencia en el tratamiento de la información y la competencia digital de los estudiantes, del mismo modo que la utilización de los lenguajes gráfico y estadístico ayuda a interpretar mejor la realidad expresada por los medios de comunicación. Es necesario, si queremos que el desarrollo tecnológico sea compatible con el de las personas, fomentar, aportando los recursos necesarios, una capacitación de las personas en ciencia y tecnología y como en ellas está implícito el conocimiento matemático resulta indispensable educar el pensamiento matemático y en particular el estadístico. En la construcción del conocimiento los medios tecnológicos son herramientas esenciales para enseñar, aprender y, en definitiva para hacer matemáticas, proponemos un método de enseñanza en el que el aprendizaje matemático tenga lugar a través de prácticas que impliquen la actividad del alumno, como son la resolución de problemas, la discusión, el establecimiento de conjeturas, etc. En este sentido estaríamos hablando más de "hacer matemáticas" que de "conocer matemáticas".

\section{Objetivos}

En el marco de la "sociedad de la información", donde los conocimientos necesarios para desarrollar la actividad profesional se renuevan continuamente, parece claro que la escuela no puede proporcionar a todos los estudiantes todos los saberes que integran la cultura actual, sobretodo en alumnos con poca regularidad asistencial a las clases y que presentan problemas de aprendizaje y de conducta. En este sentido las competencias básicas constituyen unos "mínimos" que proporcionan referencias sobre los principales aspectos donde es preciso centrar los esfuerzos, sin pretender suplantar el diseño curricular escolar para los alumnos con un rendimiento más deficitario, sino que pretenden hacer reflexionar y promover la inclusión de 
estas habilidades o aprendizajes claves en todos los currícula de la enseñanza obligatoria, pero más alla de estas competencias básicas, debemos intentar alcanzar con cada uno de nuestros estudiantes los "máximos" posibles. (Marqués, 2006).

De acuerdo con los planteamientos anteriores, la estructura de las actividades y las medidas que tomamos de atención a la diversidad están orientadas a responder a las necesidades educativas de nuestros alumnos y a conseguir las competencias y objetivos básicos marcados por el currículo correspondiente. En definitiva, pretendemos que un grupo de alumnos de diversificación curricular del IES, a través de un entorno interactivo de aprendizaje, desarrollen una serie de competencias matemáticas y destrezas básicas en la utilización de las fuentes de información, para con sentido crítico adquirir nuevos conocimientos y una preparación en el campo de las tecnologías de la información y comunicación y comprender y expresar con corrección textos y mensajes. Como objetivo de la investigación tratamos de describir, ejemplificar y analizar las bondades de una herramienta metodológica construida para el aprendizaje de la Estadística en la ESO.

\section{3. ¿Qué es y como funciona nuestro entorno de aprendizaje?}

Los elementos básicos que constituyen nuestro entorno son una página Web escrita en html, donde planteamos las actividades a realizar por nuestros alumnos, un navegador de Internet con posibilidad de manejar el correo electrónico (fundamentalmente a través de Webmail), una hoja electrónica de cálculo (en nuestro caso utilizamos Excel, pero puede ser cualquier otra) y un foro de discusión donde los alumnos y el profesor (como un alumno avanzado) se plantean cuestiones de los tópicos a resolver..

En algunos ámbitos de la enseñanza de las matemáticas, como por ejemplo en Geometría (Cabri, Sketchpad, Cinderella...), se utilizan programas informáticos pensados específicamente para el proceso de enseñanza aprendizaje, que además son interactivos y que permiten recoger el historial de la actividad del alumno durante los experimentos y el análisis de esta información, para conocer qué pasa por la cabeza de los alumnos cuando están implicados en la actividad matemática, cómo analizan y transforman la información. Aspectos fundamentales a tener en cuenta para mejorar el proceso de aprendizaje. De aquí el interés en que la recogida de la información sobre el proceso que han seguido en la resolución del problema, sea lo más completa posible ya que resulta fundamental para el investigador de cara a determinar las claves para entender qué han hecho los estudiantes y por qué lo han hecho (Gutiérrez. 2005).

En el caso de nuestra investigación la aplicación informática que hemos seleccionado es la Hoja de Cálculo, concretamente Excel, en la que no existe ningún comando o función específico que nos permita revisar los pasos que han dado los alumnos a lo largo del proceso de resolución de la actividad, pero hemos aprovechado los "recursos" de la propia hoja de cálculo para conseguirlo. Utilizando la función "compartir el Libro", tenemos la posibilidad de saber cuándo se hizo un determinado cambio, quién lo hizo y dónde (en qué celda). Estos cambios de cuándo, quién y dónde se pueden observar bien haciendo que queden resaltados en la propia pantalla de trabajo o bien recogiendo los cambios en un nueva hoja llamada Historial. El perfil de trabajo del alumno queda registrado por tanto en el Historial y como está protegido, los alumnos no pueden modificarlo ni borrarlo. A continuación ilustramos el Historial de cambios de las dos maneras citadas: 


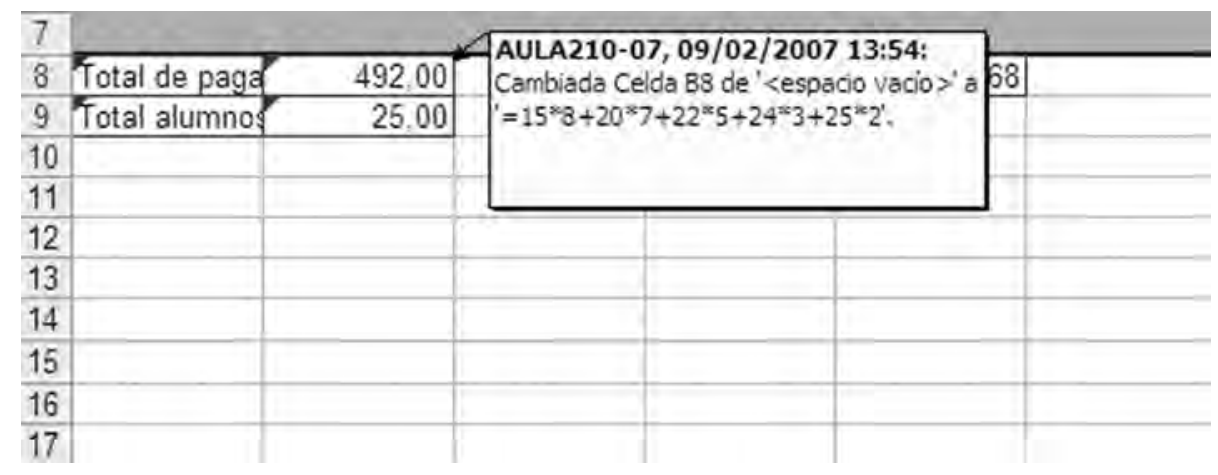

\begin{tabular}{c|c|c|c|c|c|c|c|}
\hline $\begin{array}{c}\text { Nümero } \\
\text { de } \\
\text { acción }\end{array}$ & Fecha & Hora & Quién & Cambiar & Hola & Rango & $\begin{array}{l}\text { Valor } \\
\text { nuevo }\end{array}$ \\
\hline 1 & $09 / 02 / 2007$ & $13: 54$ & AULA210-07 & Cambio de celda & Actividad & A8 & Total de paga \\
\hline 2 & $09 / 02 / 2007$ & $13: 54$ & AULA210-07 & Cambio de celda & Actividad & B8 & $=15^{*} 8+20^{*} 7+22^{*} 5+24 * 3+25 * 2$ \\
\hline 3 & $09 / 02 / 2007$ & $13: 54$ & AULA210-07 & Cambio de celda & Actividad & A9 & Total alumnos \\
\hline 4 & $09 / 02 / 2007$ & $13: 54$ & AULA210-07 & Cambio de celda & Actividad & B9 & $=8+7+5+3+2$ \\
\hline 5 & $09 / 02 / 2007$ & $13: 54$ & AULA210-07 & Cambio de celda & Actividad & D8 & Paga media \\
\hline 6 & $09 / 02 / 2007$ & $13: 55$ & AULA210-07 & Cambio de celda & Actividad & E8 & $=B 8 / B 9$ \\
\hline
\end{tabular}

El registro automático de los cambios, solo se puede establecer cada 5 minutos, por lo tanto para que se registren todos los cambios en cada una de las celdas, es necesario que el usuario guarde el fichero cada vez que lo modifica, pues en caso contrario solo se guardarían el estado inicial y final de la celda y los estadios intermedios correspondientes a los 5 minutos de actualización automática, perdiendo posiblemente el perfil de resolución de la actividad. Es fundamental para que los datos queden completamente recogidos, que los alumnos trabajen de esta manera, guardando el fichero cada vez que efectúen alguna modificación, y así poder analizar completamente el proceso de resolución.

Por otra parte, las hojas de cálculo no son programas específicamente educativos y tampoco son aplicaciones interactivas, aspectos fundamentales para trabajar en un entorno de aprendizaje interactivo, que conseguimos al integrarla en el sistema tutorial que hemos diseñado, utilizando los propios "recursos" de la hoja de cálculo y la estructura de las actividades planteadas a los alumnos. (ver Figura 1 más adelante)

\subsection{La interfaz gráfica del alumno}

Lo que el alumno verá cuando acceda al archivo de una de las actividades que se han planteado en la página Web correspondiente, es lo siguiente:

1. En un rango de celdas protegidas, establecido por el profesor, aparece el enunciado de la actividad que tiene que resolver.

2. Existe un área de trabajo para el alumno, dónde puede realizar todo tipo de operaciones que estime oportuna. Una vez obtenida una respuesta que el alumno considere adecuada la debe introducir en una celda establecida al efecto. 
3. A continuación el alumno debe elegir la respuesta del menú desplegable que más parecida sea a la que éste haya introducido y seguidamente debe guardar los cambios.

Si el alumno después de trabajar no llega a una respuesta que considere adecuada, tenemos dos opciones, la primera es que le mande el archivo al profesor; y la segunda es que le dejemos ir al paso 3 y ver las posibles respuestas y elegir una o más para ver si eso le ayuda a resolver la actividad.

4. A la izquierda del menú desplegable aparece un número (en rojo) asociado a la respuesta que aparece en pantalla. En la celda establecida se debe introducir dicho número.

5. Al introducir el número en la celda establecida (paso 4) aparecerá una "reacción-respuesta" en la celda correspondiente.

6. Si la respuesta es correcta el alumno termina, en otro caso volverá al paso 2 pero ahora con la información que le ha aportado el sistema tutorial (entorno de aprendizaje)

Se ilustra a continuación en la Figura 1 la interfaz del alumno:

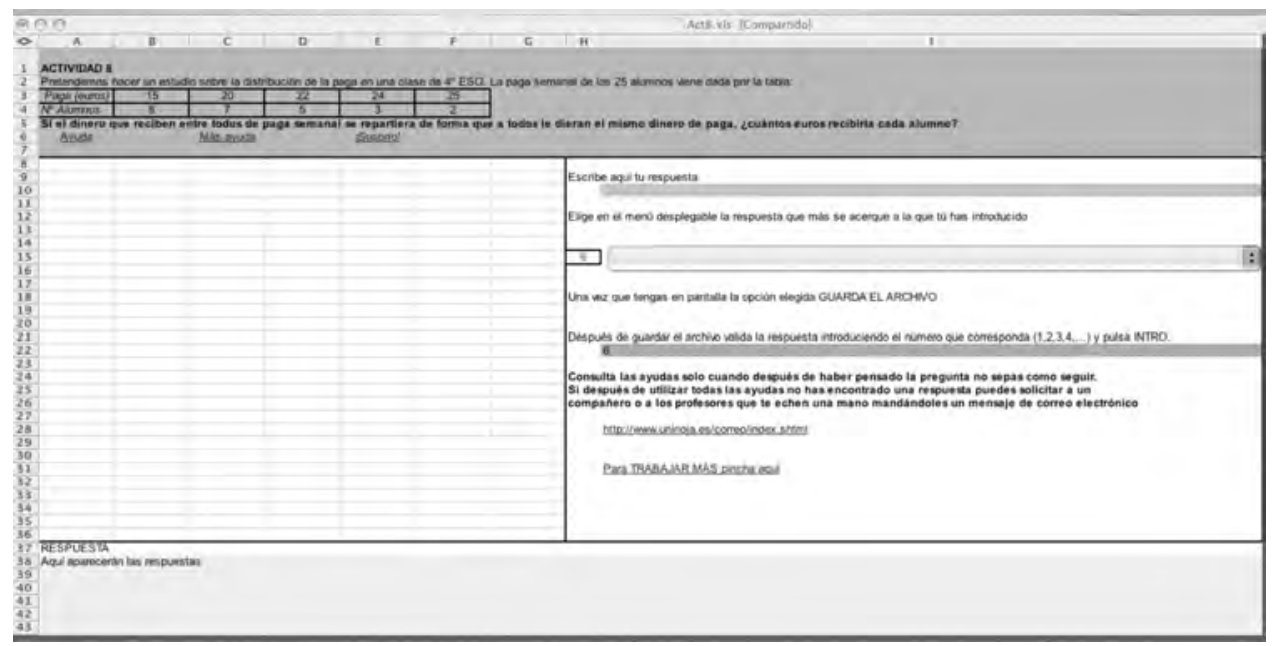

Figura 1

\subsection{La interfaz del profesor}

La interfaz del profesor aparentemente es la misma que la del alumno, sin embargo tiene acceso a cambiar el enunciado de la actividad, las ayudas y en especial en una parte oculta para el alumno puede modificar las respuestas que aparecen en el menú desplegable así como las reacciones que mostrará el sistema cuando el alumno elija esa respuesta. Hemos considerado cinco posibles respuestas (de la 1 a la 5) dejando la 6 como valor de control (inicio) para un posterior análisis de las producciones de los alumnos a través del Historial. Las celdas de introducción del número de respuesta, se han "validado" para que únicamente permitan los valores enteros del 1 al 6 ambos inclusive. Se ha restringido el área de trabajo a una porción de la Hoja1 
del libro, no pudiéndose utilizar más que esa zona. La Figura 2 sirve como ilustración.

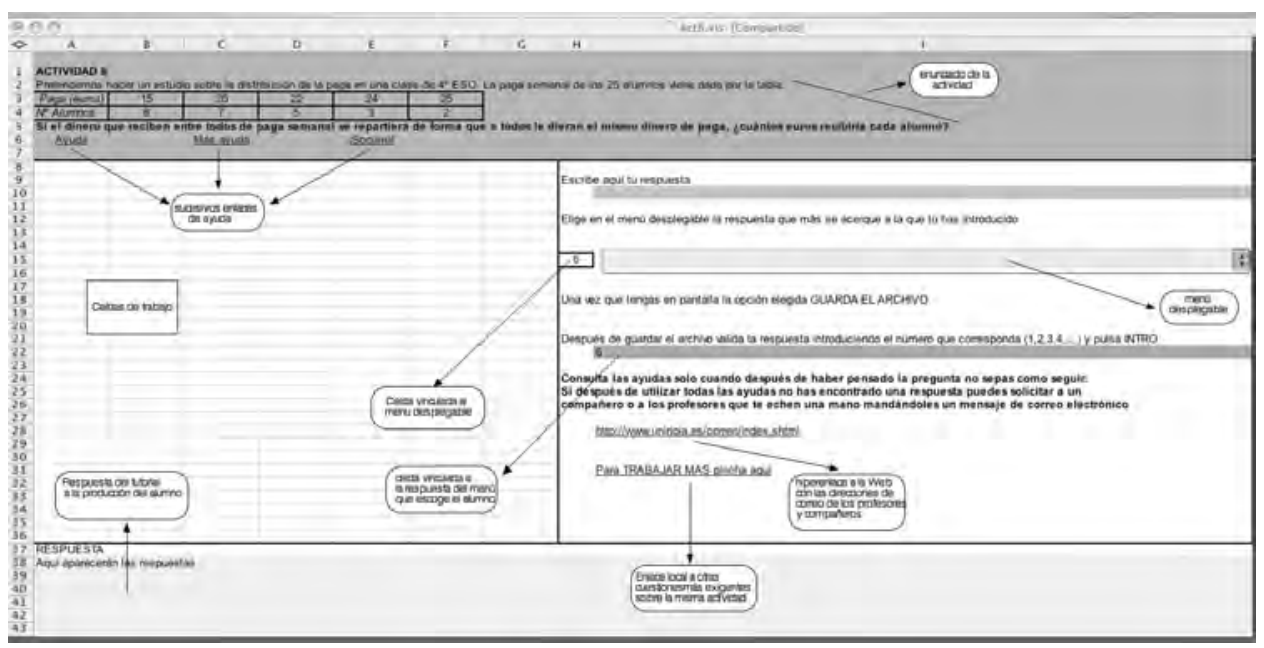

Figura 2

Sólo se admiten números con dos decimales aunque en Excel, el aspecto de un número es diferente del número almacenado en la celda. La mayoría de las veces, aunque parezca que un número se está redondeando, sólo se redondea su presentación. En los cálculos se utilizará el número que está detrás de la presentación. Es necesario que los alumnos validen sus cambios, cada vez que modifican algo, puesto que de otra forma no se grabarán esas acciones en el Historial (control de cambios) ya que lo máximo que nos permite el sistema es guardar los cambios cada 5 minutos.

\section{Metodología}

Consideramos que fundamentalmente la actividad del profesor debe ser la de moderar y guiar el trabajo y la discusión de los estudiantes, facilitando en la medida que sea posible el aprendizaje y ayudándoles a lo largo del proceso de resolución de las actividades planteadas, de manera que el discurso instruccional se convierte en un diálogo. En cuanto al alumno pretendemos conseguir una implicación activa en la adquisición del conocimiento, favorecida por el diseño de las actividades, que permiten a los alumnos participar en ellas de manera que les interesen y comprendan la finalidad de las mismas y en las que en su resolución se utilizan medios informáticos. El entorno de aprendizaje se constituye en una comunidad de aprendizaje, organización compleja en la que todos sus miembros deben asumir la responsabilidad de su aprendizaje, en función del grado de comprensión de la actividad y del papel que les corresponde.

Trabajamos en nuestra investigación con un grupo de nueve alumnos de ambos sexos del grupo de Diversificación Curricular de $4^{\circ}$ ESO de un IES de la Comunidad Autónoma de La Rioja, seis de ellos provienen de $3^{\circ}$ ESO del mismo programa y los 
tres restantes se han incorporado este año al repetir curso y juzgar su equipo docente que no debían continuar en un curso ordinario.

Cada alumno dispone de un ordenador personal conectado a Internet que le identifica unívocamente en las actividades que realiza, de forma que en el Historial quedará reflejado con el nombre asignado al ordenador (AULA210-01 hasta AULA21009). El grupo es muy heterogéneo en el sentido de que cada uno de ellos podríamos decir que es un caso de "educación especial", aunque por otro lado es homogéneo en cuanto a la edad (todos cumplirán dieciocho años este año) y en cuanto a los problemas con los estudios que todos han tenido (todos han repetido curso dos veces a lo largo de su vida académica por distintas causas). Además cuando entraron en el Programa de Diversificación tenían una media de cuatro materias pendientes de años anteriores, siendo uno de ellos un alumno con necesidades educativas especiales (ACNEE) debido a sus graves problemas de dislexia. Podríamos decir sin riesgo a equivocarnos que estos alumnos habrían abandonado el sistema educativo de no estar en el programa de Diversificación, ya que han Ilegado al segundo ciclo de la ESO más por imperativo legal, su edad, que por los conocimientos que tienen.

\subsection{Primera fase}

Desarrollamos el proyecto con seis alumnos que estaban en $3^{\circ} \mathrm{ESO}$, del programa de Diversificación para que utilizaran las Tecnologías de la Información y de la Comunicación (TIC) en el aprendizaje de las Matemáticas, aplicadas a una parte del currículo de la Estadística en la ESO. En esta fase utilizamos una primera versión de nuestro sistema tutorial interactivo. Las Matemáticas están integradas dentro del Área científico-tecnológica con un total de 6 horas semanales de las que se dedicaron al proyecto dos horas de media semanal. El proyecto se utilizó como refuerzo a la docencia personal, así además de adquirir los conocimientos en el aula según el modelo tradicional los alumnos dispusieron de una plataforma que les permitió acceder también a una acción tutorial a distancia del profesor vía la plataforma.

En esta primera fase, dadas las características de los alumnos con los que comenzamos la investigación queríamos conocer el estado general en cuanto a aptitudes diferenciales y generales, la evolución de determinados conocimientos y destrezas del currículo de la ESO en Estadística, en el aprendizaje y uso de las TIC y grado de satisfacción por el uso de nuestro entorno interactivo de aprendizaje.

Para determinar la evolución de los mismos en cuanto a aptitudes diferenciales y generales, realizamos la primera prueba del test de BADYG $^{[1]}$ (Yuste, 2000) en febrero del 2005 y la segunda en junio del 2006, una vez habían trabajado con el sistema tutorial. En la siguiente tabla recogemos los resultados comparativos.

[1] El BADYG es un test colectivo, es decir, que se realiza con todos los alumnos de la clase al mismo tiempo, Es una prueba que sirve para conocer la inteligencia de los niños en edad escolar. Lo más interesante es la "idea global del resultado", más que una prueba particular que puede estar sesgada. 
PERCENTILES (16 feb 2005/ 20 junio 2006)

\begin{tabular}{|l|c|c|c|c|c|c|c|c|c|c|c|}
\hline ALUMNOS & IG & RL & RA & SN & ML & CO & PN & EF & MA & MV & A \\
\hline Alumno 1 & $50 / 66$ & $70 / 66$ & $59 / 73$ & $38 / 23$ & $97 / 92$ & $30 / 46$ & $20 / 54$ & $59 / 77$ & $62 / 66$ & $70 / 73$ & $54 / 62$ \\
\hline Alumno 2 & $85 / 95$ & $82 / 89$ & $46 / 73$ & $89 / 82$ & $89 / 95$ & $30 / 73$ & $85 / 89$ & $95 / 98$ & $73 / 62$ & $23 / 41$ & $62 / 54$ \\
\hline Alumno 3 & $70 / 85$ & $70 / 77$ & $82 / 97$ & $66 / 54$ & $50 / 66$ & $54 / 54$ & $73 / 92$ & $66 / 87$ & $85 / 89$ & $8 / 13$ & $62 / 27$ \\
\hline Alumno 4 & $8 / 20$ & $23 / 30$ & $23 / 23$ & $38 / 23$ & $27 / 38$ & $3 / 50$ & $8 / 23$ & $18 / 38$ & $23 / 18$ & $50 / 50$ & $27 / 89$ \\
\hline Alumno 5. & $95 / 99$ & $94 / 99$ & $95 / 92$ & $92 / 98$ & $85 / 99$ & $95 / 97$ & $66 / 89$ & $89 / 95$ & $97 / 97$ & $6 / 46$ & $80 / 87$ \\
\hline Alumno 6 & $-/ 54$ & $-/ 66$ & $-/ 59$ & $-/ 23$ & $-/ 98$ & $-/ 15$ & $-/ 23$ & $-/ 85$ & $-/ 46$ & $-/ 6$ & $-/ 20$ \\
\hline
\end{tabular}

Al Alumno 6, no se le pudo hacer la prueba en febrero del 2005, por sus graves problemas de dislexia.

\section{Claves:}

\begin{tabular}{|l|l|l|}
\hline IG: Inteligencia General. & ML: Matrices Lógicas. & MA: Memoria Auditiva. \\
\hline RL: Razonamiento Lógico. & CO: Completar Oraciones. & MV: Memoria Visual. \\
\hline RA: Relaciones Analógicas. & PN: Problemas Numéricos. & A: Atención. \\
\hline SN: Series Numéricas. & EF: Encajar Figuras. & \\
\hline
\end{tabular}

Para analizar la evolución de las destrezas y habilidades hemos realizado un test inicial y otro final, tanto a los alumnos del proyecto como al resto de los alumnos de $3^{\circ}$ de ESO.

La primera prueba se pasó a los alumnos de $3^{\circ}$ ESO a principios de Diciembre de 2005, para comparar a todos los alumnos con los participantes en el Proyecto y a éstos con el resto de los de su clase ordinaria. Se realizaron 60 pruebas, 6 pertenecientes a los alumnos participantes en el proyecto y las 54 restantes pertenecientes al resto de los cursos de $3^{\circ}$ ESO. La prueba constaba de 40 ítems en los que se preguntan cuestiones relacionadas con los fundamentos de la Estadística Descriptiva unidimensional, gráficos y su interpretación, medidas de centralización y posición y medidas de dispersión. Se calificó a un punto por ítem. Hay que hacer notar que una parte importante de los ítems corresponden exclusivamente a los contenidos del currículo de $3^{\circ} \mathrm{ESO}$, por lo que todos los alumnos que provienen de $2^{\circ} \mathrm{ESO}$, es decir, que no están repitiendo $3^{\circ} \mathrm{ESO}$, no han recibido nunca hasta ahora instrucción sobre dichos conceptos por lo que era de esperar no muy buenos resultados. No obstante, se incluyeron para ver si había una diferencia significativa entre las respuestas dadas por esos alumnos y sus compañeros que están repitiendo $3^{\circ}$ ESO y por tanto ya fueron instruidos sobre estos aspectos en las clases de Matemáticas del curso pasado 2004/05.

En la última semana de clase de Junio de 2006 cuando los alumnos acabaron los exámenes correspondientes al curso académico se les volvió a pasar la misma prueba (solamente se cambiaron los valores de los datos numéricos) a los alumnos de $3^{\circ}$ 
ESO de los grupos B, C y D ( al grupo A no fue posible) con el objeto de determinar si había habido alguna diferencia importante en el nivel de conocimientos de Estadística, así como en su relación entre alumnos participantes en el proyecto y los que no participan. Se pospuso la prueba para estas fechas porque por un lado en los cursos ordinarios de Matemáticas de $3^{\circ}$ ESO la instrucción del tema de Estadística está prevista en la programación correspondiente en la última parte del curso, y por otro lado, los alumnos participantes en este proyecto siguieron trabajando en él hasta el final de curso. Una vez realizadas las pruebas de Junio se procedió a un estudio comparativo entre los resultados de antes y después de la utilización del sistema tutorial por los alumnos que han participado en este Proyecto y el resto de alumnos que ha seguido una enseñanza según el modelo tradicional.

Los resultados globales de 3D frente al resto, sobre 40 puntos de los resultados de la prueba fueron:

\begin{tabular}{|c|c|c|c|c|c|c|c|c|c|}
\cline { 2 - 10 } \multicolumn{1}{c|}{} & \multicolumn{3}{c|}{ INICIAL } & \multicolumn{3}{c|}{ FINAL } & \multicolumn{3}{c|}{ VARIACIÓN } \\
\cline { 2 - 10 } \multicolumn{1}{c|}{} & Media & D. T & C.V. & Media & D. T & C.V. & Media & D. T & C.V. \\
\hline 3D & 8,29 & 4,64 & 0,56 & 18,75 & 7,89 & 0,42 & 10,46 & 3,25 & $-0,14$ \\
\hline $3^{\text {a } A, B, C ~}$ & 6,21 & 4,09 & 0,66 & - & - & - & - & - & - \\
\hline $3^{\circ}$ B,C & 5,11 & 3,39 & 0,66 & 9,67 & 6,41 & 0,66 & 4,55 & 3,02 & 0 \\
\hline
\end{tabular}

Se observa que hay una mejora de los resultados tanto en 3D como en el resto de los grupos a lo largo del curso académico. Los resultados iniciales de los alumnos de diversificación curricular, aún siendo malos, eran algo mejores que los del resto de los alumnos, quizá debido a que había preguntas que correspondían al currículo de $3^{\circ}$ ESO y los alumnos que el año anterior cursaron $2^{\circ}$ curso y estaban este año en un curso ordinario de tercer curso no habían sido adiestrados en esos aspectos todavía, mientras que todos los alumnos del grupo de diversificación eran repetidores de tercer curso.

Sin embargo, como se puede observar, la mejoría en los resultados es mucho más acusada en 3D que en el resto de los alumnos. La desviación típica ha sido algo mayor en 3D pero el coeficiente de variación es menor por lo que en este sentido los resultados obtenidos por el grupo de diversificación son más homogéneos, es decir, están menos dispersos en relación a su resultado medio que los de todos los alumnos.

El uso del sistema tutorial interactivo, parece que produce una mejora ostensible en los resultados de los alumnos de diversificación y que sus resultados son más homogéneos.

Un aspecto a tomar en consideración, son los bajos resultados que obtuvieron en la prueba final el resto de los grupos. Alguna de las causas que se podrían apuntar, es que el tema de Estadística es de los últimos que se imparten y debido a la extensión del temario no se haya explicado suficientemente o más probablemente que el grupo de alumnos que hubo el año pasado en tercero de la ESO tenía unas bajas capacidades. No obstante hemos de decir que precisamente este tipo de alumnos son los que nutren los programas de diversificación por lo que parece que el uso del sistema tutorial interactivo es recomendable. 


\section{LOS RESULTADOS GLOBALES DE 3D FRENTE A TODOS, SOBRE 40 PUNTOS}

\begin{tabular}{|c|c|c|c|c|c|c|c|c|c|}
\cline { 2 - 9 } \multicolumn{1}{c|}{} & \multicolumn{3}{c|}{ INICIAL } & \multicolumn{3}{c|}{ FINAL } & \multicolumn{3}{c|}{ VARIACIÓN } \\
\cline { 2 - 9 } \multicolumn{1}{c|}{} & Media & D. T & C.V. & Media & D. T & C.V. & Media & D. T & C.V. \\
\hline 3D & 8,29 & 4,64 & 0,56 & 18,75 & 7,89 & 0,42 & 10,46 & 3,25 & $-0,14$ \\
\hline TODOS & 6,42 & 3,47 & 0,65 & 11,43 & 7,52 & 0,66 & 5,01 & 4,05 & 0,01 \\
\hline
\end{tabular}

A la vista de los resultados, no hay variaciones significativas en relación a los globales de 3D frente al resto de sus compañeros. Sirven las mismas reflexiones y comentarios anteriores, por lo que no añadimos nada nuevo.

Para ver con más claridad la evolución de los alumnos participantes en el Proyecto presentamos los resultados individuales de los seis alumnos de diversificación en las pruebas inicial y final.

RESULTADOS INDIVIDUALES OBTENIDOS POR LOS ALUMNOS DE DIVERSIFICACIÓN EN LA PRUEBA INICIAL (I) Y FINAL (F). 3D. CURSO 2005/2006

\begin{tabular}{|l|c|c|c|c|c|c|c|c|c|c|c|c|}
\hline Alumnos & \multicolumn{2}{|c|}{ Global } & \multicolumn{2}{c|}{ Generales } & \multicolumn{2}{c|}{ Gráficas } & \multicolumn{2}{c|}{ Centrales } & \multicolumn{2}{|c|}{ Dispersión } & \multicolumn{2}{|c|}{ Interpretación } \\
\hline & $\mathrm{I}$ & $\mathrm{F}$ & $\mathrm{I}$ & $\mathrm{F}$ & $\mathrm{I}$ & $\mathrm{F}$ & $\mathrm{I}$ & $\mathrm{F}$ & $\mathrm{I}$ & $\mathrm{F}$ & $\mathrm{I}$ & $\mathrm{F}$ \\
\hline Alumno 1 & 5,25 & 13,25 & 1,25 & 1,25 & 3 & 2 & 1 & 5 & 0 & 3 & 0 & 2 \\
\hline Alumno 2 & 8,25 & 23,25 & 1,25 & 3,25 & 3 & 8 & 0 & 4 & 0,5 & 6,5 & 3,5 & 1,5 \\
\hline Alumno 3 & 14,75 & 21,75 & 2,5 & 3,25 & 4 & 8 & 3 & 4 & 1,75 & 4,5 & 3,5 & 2 \\
\hline Alumno 4 & 8,5 & 8,25 & 0 & 2 & 2 & 3 & 2,5 & 1 & 1,5 & 1,25 & 2,5 & 1 \\
\hline Alumno 5 & 1,5 & 15,75 & 0,5 & 2,75 & 0 & 5 & 0 & 4 & 0 & 3 & 1 & 1 \\
\hline Alumno 6 & 11,5 & 30,25 & 2 & 3,75 & 2 & 8 & 3 & 6 & 0 & 7 & 4,5 & 5,5 \\
\hline
\end{tabular}

$\square$ Alumno1 $\square$ Alumno2 $\square$ Alumno3 $\square$ Alumno4 $\square$ Alumno5 $\square$ Alumno6

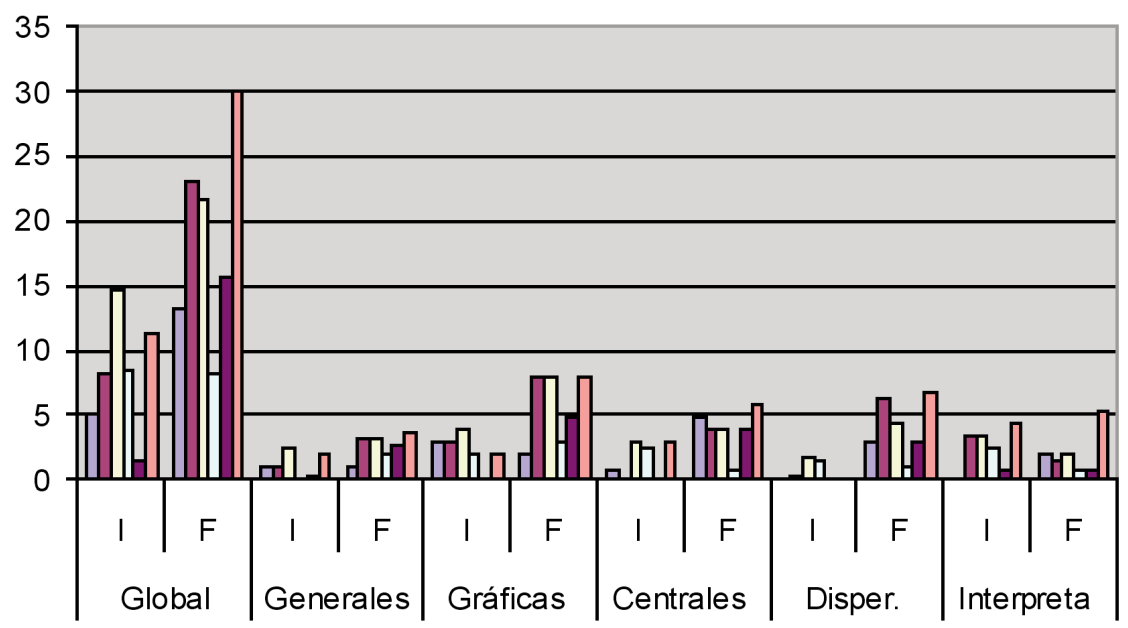




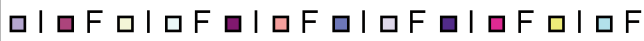

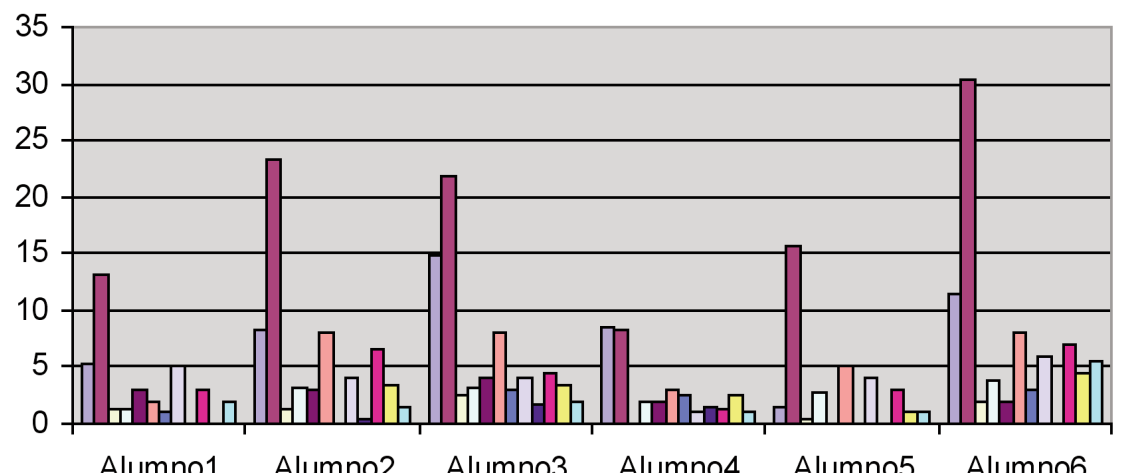

Se observa que todos mejoran en sus resultados globales, con la excepción del Alumno4 (concuerda con los resultados bajos que obtiene en el test de BADYG-r en el apartado de Memoria Visual que mide entre otras cosas la retentiva a largo plazo). El aumento en la puntuación media del resto es apreciable. En especial destaca la mejora global del Alumno2, que a pesar de estar diagnosticado como ACNEE desde la educación primaria debido a su severa dislexia ha obtenido en la prueba final la segunda mejor nota.

\section{Grado de satisfacción de los participantes en el Proyecto}

El grado de satisfacción de los alumnos participantes en el Proyecto en relación al uso de este nuevo método de enseñanza, lo analizamos a través de una encuesta anónima.

\subsubsection{Modelo de la encuesta pasada}

Cada uno de los ítems utilizados se podía valorar como:

Nada. b) Poco. c) Regular. d) Bastante. e) Mucho.

\section{ENCUESTA}

1. ¿Te ha parecido interesante esta experiencia?

2. ¿Asistes con regularidad a estas clases?

3. ¿Asistes a gusto a estas clases?

4. ¿Encuentras satisfactorio el trabajo que has realizado en estas clases?

5. ¿Estás satisfecho con el trabajo llevado a cabo por los profesores?

6. ¿Crees que los contenidos impartidos en estas clases son importantes para tu formación académica?

7. ¿Crees que lo que has aprendido te puede servir en tu futuro laboral?

8. ¿Crees que las actividades planteadas en la página Web y en el foro de discusión son adecuadas a vuestros conocimientos de Estadística? 
9. ¿Las actividades están planteadas de forma clara?

10. Las respuestas dadas por los profesores a través del correo electrónico y del foro de discusión, ¿han sido efectuadas de forma clara y precisa?

11. ¿Los profesores se interesan por el aprendizaje de sus alumnos?

12. La atención recibida por parte del profesor presencial y del profesor virtual en las consultas que les has hecho, iha sido la adecuada?

13. ¿Te ha gustado este nuevo enfoque del estudio de las Matemáticas a través del ordenador e Internet?

14. ¿Te interesaría participar de nuevo en una experiencia de este tipo?

15. Considerando globalmente todos los aspectos de estas clases, ¿estás satisfecho con el desarrollo de las mismas?

16. ¿Te ha resultado gratificante participar en esta experiencia?

17. Valora el grado de aplicación de una experiencia semejante en el estudio de estas otras materias de $3^{\circ} \mathrm{ESO}$ (marca con una $\mathrm{x}$ ):

\begin{tabular}{|l|l|l|l|l|l|}
\hline Materias & Nada & Poco & Regular & Bastante & Mucho \\
\hline Física y Química & & & & & \\
\hline Ciencias Sociales & & & & & \\
\hline Biología y Geología & & & & & \\
\hline Educación Plástica & & & & & \\
\hline Tecnología & & & & & \\
\hline Lengua Española & & & & & \\
\hline Inglés & & & & & \\
\hline Francés & & & & & \\
\hline
\end{tabular}

Si lo consideras necesario escribe lo que creas oportuno acerca de la experiencia que hemos realizado:

\subsubsection{Resultados de la encuesta de satisfacción}

La mayoría de los participantes han valorado la mayoría de los ítems con Bastante o Mucho. De hecho las respuestas a las preguntas 1, 2, 3, 4, 5, 10, 11, 12, 13, y 15 han sido valoradas por el $100 \%$ de los alumnos con la máxima valoración de Bastante o Mucho. Las respuestas a las preguntas 6, 7, 8, 9, y 14 han sido valorados por el $83 \%$ de los alumnos con la máxima valoración de Bastante o Mucho. En cuanto a la pregunta 17 en la que se preguntaba qué grado de aplicabilidad tendría un proyecto semejante al que hemos realizado en Matemáticas en el resto de las materias de $3^{\circ} \mathrm{ESO}$ las respuestas de los alumnos varían bastante dependiendo de que la materia sea de tipo científico o de tipo no científico, valorando en su opinión el grado de aplicabilidad de las primeras más positivamente que de las segundas. Valoramos especialmente la respuesta a la pregunta 7 , en las que el $83 \%$ la valora con las máximas puntuaciones de Bastante o Mucho, lo que hoy en día es un especial tanto a favor puesto que una de las habituales quejas de los alumnos es que lo 
que se estudia en los Institutos luego "no sirve para nada", es decir que no lo van a utilizar luego en su futuro laboral.

Así mismo es de destacar la respuesta a la pregunta 13, en las que el 100\% la valora con las máximas puntuaciones de Bastante o Mucho lo que muestra el grado de interés suscitado por esta experiencia en los alumnos.

Finalmente, como resumen del grado de satisfacción de los alumnos en relación a la utilización del sistema tutorial, hemos escogido una de las respuestas dada por uno de los alumnos a la número 18, que era de respuesta libre: "Esta actividad merece mucho la pena, y además en el futuro nos va a servir de gran ayuda, porque el ordenador es el futuro".

\subsection{Segunda fase}

Desde una visión constructivista del aprendizaje, los alumnos deben participar de forma activa en su aprendizaje, facilitamos esta participación usamos un entorno de aprendizaje interactivo constituido por una red electrónica, Internet, software de correo y de navegación de dominio público y una hoja de cálculo electrónica Excel. Sin embargo, las hojas de cálculo no son programas específicamente educativos y tampoco son aplicaciones interactivas, estos dos aspectos, fundamentales para trabajar en un entorno de aprendizaje interactivo se consiguen al integrarlos en el sistema tutorial que hemos diseñado, utilizando los propios "recursos" de la hoja de cálculo y la estructura de las actividades planteadas a los alumnos.

La interactividad se produce fundamentalmente por el diseño de las actividades propuestas, que responden básicamente a la siguiente estructura:

1. Enunciado de la actividad. Si el alumno no sabe como realizar la actividad, una vez que ha abierto el archivo correspondiente, en la misma página aparecen tres enlaces-ayuda progresiva, que hemos convenido en Ilamar "Ayuda" "Más Ayuda" y "Socorro" A primera vista el llamar "Socorro" al último enlace de ayuda podría parecer poco serio, sin embargo hemos considerado que introducir algo humorístico puede crear un entorno de aprendizaje positivo y relajante, reduciendo el estrés del alumno (Friedman et al, 2002). Si después de haber consultado todas las ayudas no consigue encontrar la respuesta, puede utilizar otro enlace que también aparece en la página de la actividad y en la que aparece el listado de todos los miembros del proyecto (compañeros y profesor), para solicitar en su caso, algún tipo de aclaración o ayuda.

2. Una vez que ha encontrado la respuesta o solución esta se valida.

3. Según el tipo de actividad, una vez encontrada la respuesta a la primera cuestión, en un enlace señalado como "Mas trabajo" al alumno se le van planteando cuestiones más exigentes en relación al enunciado original de la actividad. En cada ampliación de la actividad, se da la opción al alumno de consultar nuevas ayudas.

En las primeras clases se trabaja con los alumnos en el manejo del entorno interactivo y del software correspondiente. Debido a que tenemos en clase a alumnos que están familiarizados con el entorno y a otros que lo desconocen por completo y con el fin de atender a la diversidad del alumnado, mientras se les explica nuevos alumnos el manejo del entorno y los elementos de Excel que se necesitan, los demás pro- 
fundizan en el manejo de Excel, utilizando elementos del software que no se necesitarán propiamente en el proyecto.

Una vez que todos los alumnos han adquirido la destreza suficiente en el entorno y en Excel se les explica en una clase el funcionamiento de la hoja electrónica modificada por los investigadores.

\section{Conclusiones y aportaciones}

Sin estar del todo finalizada la investigación, dado que en un próximo estudio se procederá a la organización de los datos recogidos, al diseño de los correspondientes instrumentos de análisis y al análisis de los beneficios que el entorno tutorial diseñado produce en nuestros alumnos, mediante un estudio de casos, en una primera aproximación podemos establecer las siguientes conclusiones y aportaciones:

1. Hemos diseñado, descrito y ejemplificado una herramienta metodológica, construida para el aprendizaje de la Estadística en la ESO.

2. Esta herramienta metodológica, entorno interactivo para el aprendizaje de la Estadística, cuenta con unas actividades, en cuyo diseño y estructura, se ha tenido muy en cuenta, por una parte las características de nuestros alumnos, y por otra la incorporación progresiva de ayudas, planteando también propuestas de distintos niveles de exigencia en torno al problema o actividad inicial planteada. El planteamiento anterior, ha resultado fundamental para responder a las necesidades educativas de nuestros alumnos y para conseguir las competencias y objetivos básicos marcados por el currículo correspondiente.

3. La utilización efectiva del navegador de Internet y del correo electrónico, en el intercambio de respuestas y comunicaciones entre profesor y alumnos, muestra la consecución del objetivo de una preparación básica en el campo de las tecnologías de la información y comunicación.

4. El análisis de los resultados iniciales y finales, muestra que la metodología utilizada, a través de la herramienta metodológica en la que el profesor ha actuado fundamentalmente como moderador y facilitador del aprendizaje, en la que el discurso instruccional ha sido un diálogo entre profesor y alumnos y con la participación activa de los alumnos en la adquisición del conocimiento, ha proporcionado resultados globales mejorados de forma apreciable para todos los alumnos, incluido el alumno ACNE. Podemos considerar por tanto como aportación este procedimiento de enseñanza aprendizaje.

5. Los resultados de la encuesta de satisfacción muestran claramente que la utilización de esta herramienta metodológica constituye un elemento motivador para el aprendizaje.

\section{BIBLIOGRAFÍA}

BATANERO, C. (2002). Conferencia inaugural Jornadas Interamericanas de Enseñanza de la Estadística. Buenos Aires.

FORTUNY, J. M.; RICHARD, P. (2004). Distance Education at Secondary Levels: Contexts and Norms for the Learning of Mathematics . Comunicación. World Conference of Association for the Advancement of Computing in Education (ELearn Washington (USA). 04/10/2004 - 04/10/2004. 
FRIEDMAN, H.H. et al (2002). Using humor in the introductory statistics course. Journal of Statistics Education. Volume 10, Number 3.

GUTIERREZ, A. (2005). Aspectos metodológicos de la investigación sobre el aprendizaje de la demostración mediante exploraciones con software de Geometría dinámica. Investigación en Educación Matemática, $9^{\circ}$ Simposio de la SEIEM, Universidad de Córdoba.

MARQUES, P. (2006). Nuevas competencias para los ciudadanos. Departamento de pedagogía aplicada. UAB.

MURILLO, J y MARCOS, G. (2005). Un modelo de análisis de competencias matemáticas en un entorno interactivo. Investigación en Educación Matemática, $9^{\circ}$ Simposio de la SEIEM, Universidad de Córdoba.

RUMSEY, D.J. (2002). Statistical literacy as a goal for introductory statistics courses. Journal of Statistics Education. Volume 10, Number 3.

YUSTE, C y otros. (2000). Badyg-M: manual técnico. Madrid: CEPE.

RICO ROMERO, L. (2003) Evaluación de competencias matemáticas. Proyecto Pisa/OCDE 2003 en Castro, E. y De La Torre, E. (eds.), Investigación en Educación Matemática, $8^{\circ}$ Simposio de la SEIEM, Universidade da Coruña. 